\title{
Efficient techniques for BEM rank-deficiency electrostatic problems
}

\author{
Shiang-Woei Chyuan ${ }^{\mathrm{a}, *}$, Yunn-Shiuan Liao ${ }^{\mathrm{b}}$, Jeng-Tzong Chen ${ }^{\mathrm{c}}$ \\ ${ }^{a}$ Chung Shan Institute of Science and Technology, P.O. Box 90008-15-3, Lung-Tan, Tao-Yuan 325, Taiwan, ROC \\ ${ }^{\mathrm{b}}$ Department of Mechanical Engineering, National Taiwan University, Taipei, Taiwan, ROC \\ ${ }^{\mathrm{c}}$ Department of Harbor and River Engineering, National Taiwan Ocean University, Keelung, Taiwan, ROC
}

Received 22 October 2006; accepted 12 June 2007

Available online 16 July 2007

\begin{abstract}
For modern MEMS and electron devices, an accurate electrostatic analysis is essential and indispensable for engineers. The BEM is a widely used computational technique nowadays for MEMS and EM because of its superiority for unlimited exterior field. But for electrostatic problems with some specific geometry, the singularity caused by a degenerate scale will be encountered since the influence matrix is rank deficient, and numerical results become unstable. Therefore, the approach to correctly and efficiently solve the singularity arising from degenerate scale becomes a very essential and indispensable task for engineers. In this article, some efficient regularization BEM, RBM, CHIEF and hypersingular formulation, in conjunction with SVD technique, are employed to study and cope with the rankdeficiency problem numerically. These regularization techniques are successfully applied to overcome the degenerate scale and the error is suppressed in the numerical experiment.
\end{abstract}

(C) 2007 Elsevier B.V. All rights reserved.

Keywords: Singularity; Electrostatic; BEM; SVD; Regularization techniques; Degenerate scale

\section{Introduction}

Electrostatics, as used here, involves charges in motion as well as at rest. There are five fundamental quantities (voltage, charge, current, capacitance and resistance) in electrostatics, which are involved in almost all applications. For most electrical engineers, voltage, or electromotive force (EMF) is the most important one. When device dimensions are much less than the wavelength of electromagnetic radiation at a particular frequency, then the response of the system at that frequency can be considered quasistatic in that the emission, transmission, or absorption of electromagnetic radiation can be ignored [1]. Therefore, electrostatics generally plays a very important role to improve the performance and reliability of microelectromechanical systems (MEMS) and electron devices in the design stage, and many numerical methods (e.g., finite difference method, variational methods, moment methods, finite element method, boundary element method, transmis-

\footnotetext{
${ }^{*}$ Corresponding author.

E-mail address: swchyuan@ntu.edu.tw (S.-W. Chyuan).
}

sion-line-matrix method, Monte-Carlo method) were regularly used [2,3]. Among diverse numerical approaches, finite element method (FEM) [4] and boundary element method (BEM) [5] become powerful design tools for engineers because of the increasing developments of digital computer power. The FEM is one of the most widely used computational techniques because it can model extremely complex configurations and easily determine the response at any desired point of a structure. Comparatively, the BEM has a few more advantages. For example, it is easy to mesh and apply adaptive error control techniques to apply it, and its efficiency is much higher than the FEMs when facing the unbounded domain [6]. In reality, it is almost impossible to measure the field distribution inside and outside of many MEMS and electron devices, and there is no doubt that BEM has become a very popular approach in numerical simulation of electromagnetics (EM) and MEMS nowadays [7]. For most electrostatics except degenerate problems (e.g., degenerate boundary, degenerate scale), the formulation of a singular boundary integral equation for the primary field (e.g., potential, voltage) provides sufficient conditions to ensure a unique solution. While using the 
conventional BEM without laborious artificial boundary technique for electrostatic problems that have singularity due to degenerate boundaries, the coincidence of the boundaries gives rise to an ill-conditioned problem. For the singularity arising from degenerate boundaries, the dual BEM (DBEM) uses a dual integral formulation with a hypersingular integral can solve this singular problem very well, and the BEM was reformulated in terms of displacement and traction boundary integral equations by Chen and Hong [8] who thus presented the theoretical basis of dual BEM.

Besides degenerate boundary, degenerate scale is another degenerate source for singularity of BEM. For problems with special scale of geometry shape, many researchers have found that the influence matrix of the weakly singular kernel may be singular for the Dirichlet problem $[9,10]$. This non-unique solution is not physically realizable but stems from the zero eigenvalue imbedded in the influence matrix of the discrete system using the BEM formulations, and the special geometry which results in a non-unique solution for a potential problem is called degenerate scale [11]. While facing the special geometry, which results in a non-unique solution for an electrostatic problem, conventional BEM results become erratic since the influence matrix is rank deficient. Therefore, how to correctly and efficiently solve the singularity arising from degenerate scale becomes essential for engineers.

In this article, several efficient regularization techniques, rigid body mode (RBM) [12], combined Helmholtz interior integral equation formulation (CHIEF) [13] or combined Helmholtz exterior integral equation formulation (CHEEF) [14], were used to successfully overcome the non-unique solutions in numerical implementation. Using $\mathrm{RBM}$, a rigid-body term was superimposed in the fundamental solution to sort out the problem, but this approach results in a new degenerate scale instead of the original one. For CHIEF or CHEEF method, the independent constraints are added for promoting the rank of singular matrix, and the technique of singular value decomposition (SVD) was used to solve the over-determined set of linear equations [15]. Also, the role of hypersingular boundary integral equation is examined for the degenerate scale problems.

To our best knowledge, it is very important to utilize SVD technique first to check the singular status of influence matrices before the BEM was used. If the influence matrix $[U]$ of singular boundary integral equation is non-singular, either BEM or DBEM can be used, otherwise the aforementioned regularization techniques are needed. To prove this, an electrostatic problem was analyzed to check the mathematical model's validity; the analysis also showed that the ill-conditioned singular problem of BEM can be avoided and a more accurate and reasonable result can be obtained. Hence, it is strongly recommended to apply these regularization techniques for industrial applications while facing the singularity arising from degenerate scale.

\section{Integral formulations of BEM and DBEM}

The electrostatic problem consists of finding the unknown potential function $\Phi$ (or $V$ ) in the partial differential equation. In addition to the fact that $\Phi$ satisfies $\nabla^{2} \Phi=0$ within a prescribed solution region $\Omega$, the potential function $\Phi$ must satisfy certain conditions on $B$ which is the boundary of $\Omega$. Usually these boundary conditions are the Dirichlet $(\Phi(x)=f(x))$ and Neumann $\left(\partial \Phi(x) / \partial n_{x}=g(x)\right)$ types. Therefore, the governing equation of electrostatic problems could be written in the following form:

$\nabla^{2} \Phi(x)=0$,

where $f(x)$ and $g(x)$ denote known boundary data, and $n_{x}$ is the unit outer normal vector at the point $x$ on the boundary $B$.

Based on the dual boundary integral equation formulation for electrostatic problem [8], we have

$$
\begin{aligned}
\alpha \Phi(x)= & \operatorname{CPV} \int_{B} T(s, x) \Phi(s) \mathrm{d} B(x) \\
- & \operatorname{RPV} \int_{B} U(s, x)\left[\frac{\partial \Phi(s)}{\partial n_{s}}\right] \mathrm{d} B(s), \\
\alpha\left[\frac{\partial \Phi(x)}{\partial n_{x}}\right]= & \operatorname{HPV} \int_{B} M(s, x) \Phi(s) \mathrm{d} B(s) \\
& -\operatorname{CPV} \int_{B} L(s, x)\left[\frac{\partial \Phi(s)}{\partial n_{s}}\right] \mathrm{d} B(s),
\end{aligned}
$$

where the kernel functions, $U(s, x)=\ln (r), T(s, x)=$ $\partial U(s, x) / \partial n_{s}, L(s, x)=\partial U(s, x) / \partial n_{x}, \quad M(s, x)=\partial^{2} U(s, x) /$ $\partial n_{x} \partial n_{s}, r=|s-x|, s$ and $x$ being position vectors of the points $s$ and $x$, respectively, and $n_{s}$ is the unit outer normal vector at point $s$ on the boundary (see Fig. 1). Table 1 shows the explicit forms of the four kernel functions

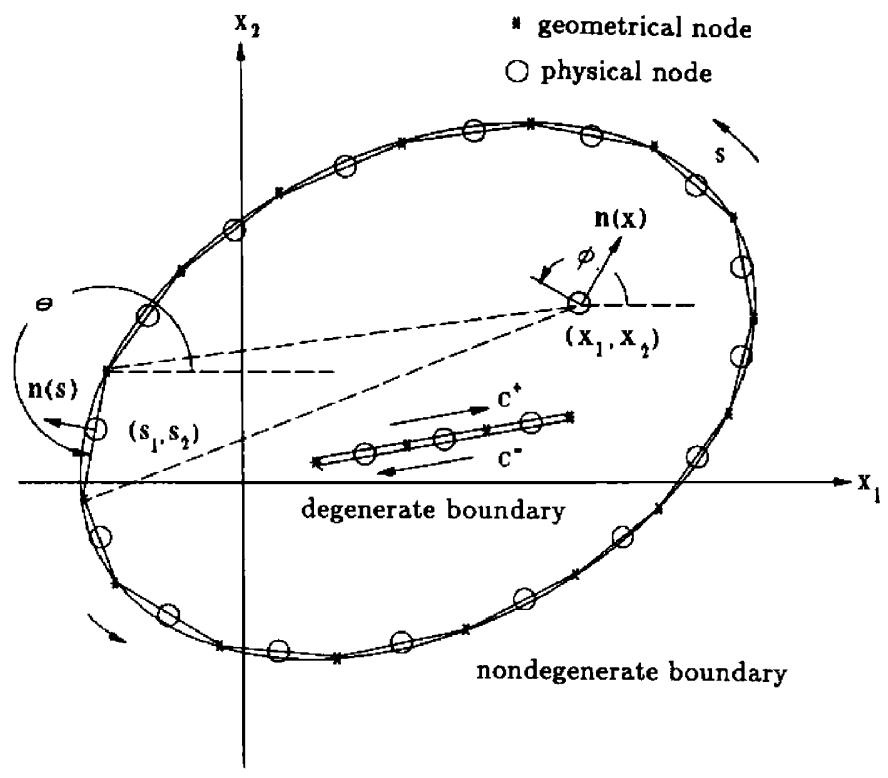

Fig. 1. Boundary element discretization for degenerate boundary and nondegenerate boundary. 
Table 1

The explicit forms of four kernel functions in dual integral equations

\begin{tabular}{lllll}
\hline Kernel function & $U(s, x)$ & $T(s, x)$ & $L(s, x)$ & $M(s, x)$ \\
\hline $\begin{array}{l}\text { Order of } \\
\text { singularity }\end{array}$ & Weak & Strong & Strong & Hypersingular \\
$\begin{array}{l}\text { Two-dimensional } \\
\text { case }\end{array}$ & $\ln (r)$ & $-y_{i} n_{i} / r^{2}$ & $y_{i} \bar{n}_{i} / r^{2}$ & $2 y_{i} y_{j} n_{i} \bar{n}_{j} / r^{4}-n_{i} \bar{n}_{i} / r^{2}$ \\
$\begin{array}{l}\text { Three-dimensional } \\
\text { case }\end{array}$ & $-1 / r$ & $-y_{i} n_{i} / r^{3}$ & $y_{i} \bar{n}_{i} / r^{3}$ & $3 y_{i} y_{j} n_{i} \bar{n}_{j} / r^{5}-n_{i} \bar{n}_{i} / r^{3}$ \\
$\begin{array}{l}\text { Remark } \\
\text { Remark }\end{array}$ & $r^{2}=y_{i} y_{i}$ & $n_{i}=n_{i}(s)$ & $\bar{n}_{i}=n_{i}(x)$ & $y_{i}=x_{i}-s_{i}$ \\
\hline & & & &
\end{tabular}

$U(s, x), T(s, x), L(s, x)$ and $M(s, x)$. In addition, RPV is the Riemann principal value, $\mathrm{CPV}$ is the Cauchy principal value, HPV is the Hadamard principal value, and $\alpha$ depends on the collocation point ( $\alpha=2 \pi$ for an interior point, $\alpha=\pi$ for a smooth boundary, $\alpha=0$ for an exterior point). The commutativity property of the trace operator and the normal derivative operator provides us with alternative ways to calculate the Hadamard principal value analytically. In the derivation of dual equations, two alternatives can be applied to determine the Hadamard principal value as shown in Fig. 2. In Fig. 2, two ways to derive Eq. (3) can be considered. One is trace operator first and then the differential operator. The other is differential first and then trace process. It must be taken care for the boundary term for the first approach while the second approach needs the $l^{\prime}$ hospital's rule.

Generally, Eq. (2) is called singular boundary integral equation, and Eq. (3) is called hypersingular boundary integral equation. Since the hypersingular boundary integral equation plays an important role in the degenerate problems, many researchers have paid much attention to this. After discretizing the boundary into $2 N$ boundary elements, Eqs. (2) and (3) are reduced to

$[U]_{2 N \times 2 N}\{t\}_{2 N \times 1}=[T]_{2 N \times 2 N}\{u\}_{2 N \times 1}$,

$[L]_{2 N \times 2 N}\{t\}_{2 N \times 1}=[M]_{2 N \times 2 N}\{u\}_{2 N \times 1}$,

where $[U],[T],[L]$ and $[M]$ are the four influence matrices, $\{u\}$ and $\{t\}$ are the boundary data for the primary and the secondary boundary variables, respectively. Generally for electrostatic problems without degenerate scale, the aforementioned influence matrix $[U]$ is nonsingular, either Eq. (4) or (5) can be solved by Gaussian elimination and LU decomposition very well. But for degenerate scale problem, $[U]$ matrix is singular and the rank is deficient, then the following SVD technique is needed.

\section{Review of the technique of singular value decomposition (SVD)}

\subsection{Basis of $S V D$}

There exists a very powerful set of techniques for dealing with sets of equations or matrices that are either singular or else numerically very close to singular. In many cases where Gaussian elimination and LU decomposition fail to give

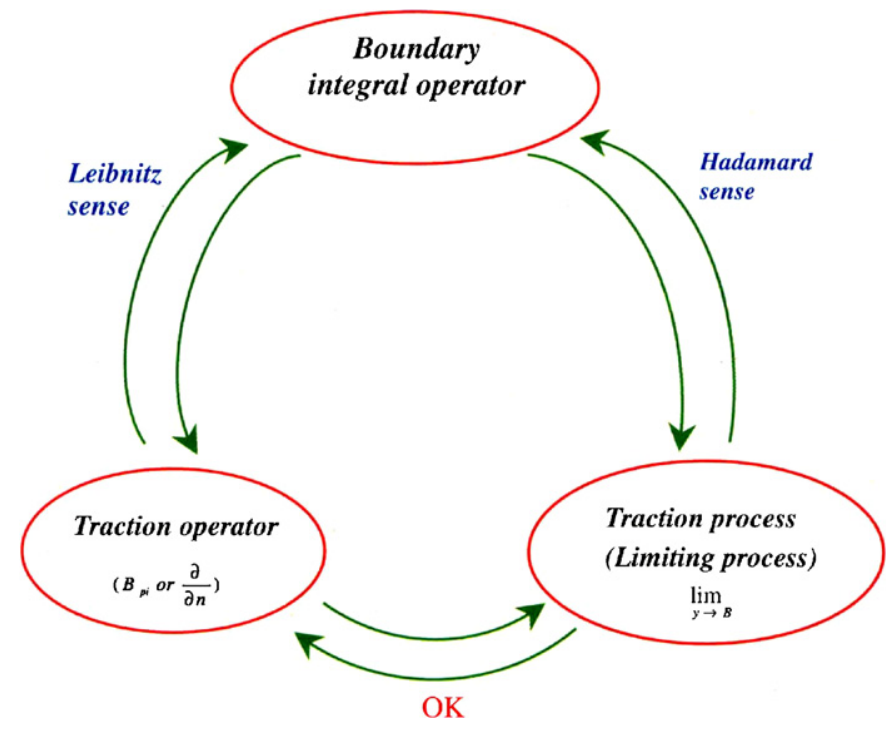

Fig. 2. Commutativity of trace and differential operator.

satisfactory results, this set of techniques, known as singular value decomposition, or SVD, will diagnose precisely what the problem is [15].

SVD methods are based on the following theorem of linear algebra: Any $M \times N$ matrix $A$ whose number of rows $M$ is greater than or equal to its number of columns $N$, can be written as the product of an $M \times N$ column-orthogonal matrix $B$, an $N \times N$ diagonal matrix $S$ with positive or zero elements (the singular values $s$ ), and the transpose of an $N \times N$ orthogonal matrix $W$ :

$[A]_{M \times N}=[B]_{M \times N}[S]_{N \times N}[W]_{N \times N}^{T}$.

\subsection{Condition number}

The condition number $c$ of a matrix $[A]$ is defined as the ratio of the largest singular value (in magnitude) of the $s_{\max }$ to the smallest of the $s_{\min }$. Generally, a matrix is singular if its condition number is infinite, and it is ill-conditioned if its condition number is too large, that is, if its reciprocal $c^{-1}$ approaches the machine's floating-point precision.

\subsection{The singularity of degenerate scale and the BEM}

Harrington et al. [10] had stated that the electrostatic problem became mathematically indeterminate for a circle of unit radius. In addition, the non-unique solution of a unit circle was also noted by other researchers [16]. Basically, the special geometry, which results in rank deficiency for a potential problem is called 'degenerate scale'. The term 'scale' stems from the fact that the numerical instability of a unit circle of radius $1 \mathrm{~m}(1 \mathrm{~cm})$ disappears if the radius of $100 \mathrm{~cm}(0.01 \mathrm{~m})$ is used in the BEM implementation. In real BEM simulation, the number one for the circular radius using the normalized scale should be avoided. Besides unit circle, the degenerate scale problem under different contours with specific 
geometry may be encountered, and the accurate degenerate scale is dependent on the discretization boundary density in BEM [17]. Therefore, it is not trivial to seek a unified method for dealing with the degenerate scale problem.

\subsection{Checking the singular status of influence matrices}

Though the singularity arising from degenerate scale is a serious problem for BEM, it is not necessary to feel concern about it if the SVD technique was efficiently and correctly used to check the singular status of influence matrix matrices [18]. For some electrostatic problems with special geometry, the $[U]$ matrix of singular boundary integral equation may be either singular or numerically very close to singular if the SVD updating technique was used to diagnose it. If $[U]$ matrix is singular, the following efficient regularization techniques for rank-deficiency problem can be used.

\section{Efficient regularization techniques for rank-deficiency problem}

In order to deal with the singularity arising from degenerate scale, several efficient regularization techniques were used as follows.

\subsection{Method of adding a rigid-body mode (RBM)}

The concept of RBM has been used in the BEM for determining the diagonal elements of the influence matrix for many years. In 1990, Vable described that the issue of RBM made the BEM less sensitive to errors or changes in the input data and arose as a consequence of implementing an algorithm. The importance of RBM which affects the BEM analysis was also described [12]. Because the nontrivial solution for the singular matrix is found to be a rigid-body term (this is both physically and mathematical realizable since RBMs are imbedded in the zero-eigenvalue matrix), the method of adding a RBM can be used to remove the RBM from the discretized linear system of degenerate scale problem. Since the $[U]$ matrix of singular boundary integral equation (Eq. (4)) is singular in case of the degenerate scale, the modified fundamental solution can be added by a rigid-body term $\beta$,

$U^{*}(s, x)=U(s, x)+\beta$.

The influence matrix $[U]$ is modified to $\left[U^{*}\right]$, and the zero singular value in $[U]$ moves to a nonzero value for $\left[U^{*}\right]$. To demonstrate the effectiveness, the condition number and minimum singular value of $\left[U^{*}\right]$ of the modified fundamental solution will be shown in the numerical example by SVD. Although the degenerate scale problem is circumvented for the special geometry, the degenerate scale moves to another size in reality.

\subsection{CHIEF or CHEEF method}

Generally, the nonuniqueness problem is numerically manifested in a rank deficiency of the BEM coefficient matrix. In order to obtain the unique solution that is known to exist analytically, several modified integral equation formulations that provide additional constraints to the original system of equations have been proposed. Schenck used the CHIEF method for exterior problem, which employs the boundary integral equations by collocating the interior point as an additional equation to make up deficient constraint condition in 1968 [13]. If the chosen point is the node of the associated interior problem, then this CHIEF method may fail. Though many researchers have enhanced and modified the CHIEF method for interior problem, the main disadvantage of the CHIEF method lies in how the number of interior points is selected and where the position should be considered. To overcome this problem, Chen et al. [14] proposed the CHEEF method in 2001. Based on the CHEEF method, the spurious solutions can be filtered out if additional constraints from the exterior points are chosen.

Although the CHIEF and CHEEF methods were first used to overcome fictitious frequency and spurious eigenvalues for radiation problem and eigenequation, respectively, these methods are extended to solve the singularity arising from degenerate scale problem in this article because the source of these two problems are both rank-deficiency. Since the rank of $[U]$ matrix for degenerate scale problem is deficient, the independent constraint is required in order to promote the rank. To resort to the null field equation by collocating the point inside (CHIEF for exterior problem) or outside (CHEEF for interior problem) the domain, we have

$\left[w^{U}\right]_{1 \times 2 N}\{t\}_{2 N \times 1}=\left[w^{T}\right]_{1 \times 2 N}\{u\}_{2 N \times 1}$,

where $\left\{w^{U}\right\}$ and $\left\{w^{T}\right\}$ are the influence row vectors by collocating the CHIEF or CHEEF point in the null-field equation. Combining Eqs. (4) and (8) yields

$\left[\begin{array}{c}{[U]_{2 N \times 2 N}} \\ \left\{w^{U}\right\}_{1 \times 2 N}\end{array}\right]\{t\}_{2 N \times 1}=\left[\begin{array}{c}{[T]_{2 N \times 2 N}} \\ \left\{w^{T}\right\}_{1 \times 2 N}\end{array}\right]\{u\}_{2 N \times 1}$.

Because conventional techniques of Gaussian elimination and LU decomposition cannot successfully solve the aforementioned over-determined set of linear equations such as Eq. (9), the reasonable solution by using SVD technique can be obtained. In addition, the condition number and minimum singular value of $[U]$ by using CHIEF or CHEEF method will also be shown in the numerical example by SVD for demonstrating its effectiveness.

\subsection{Hypersingular formulation}

By way of SVD, the condition number and minimum singular value of $[L]$ matrix can be calculated, and $[L]$ 
matrix may be nonsingular for degenerate problems if the hypersingular boundary integral equation was chosen. Instead of using the singular boundary integral equation (Eq. (4)) in the conventional BEM, the hypersingular boundary integral equation (Eq. (5)) can be used efficiently.

\section{Solving electrostatic problems with degenerate scale by the regularization techniques}

For the nondegenerate scale problems, either the conventional BEM or DBEM can be chosen. But for electrostatic problems with singularity arising from degenerate scale, the aforementioned regularization techniques play important and efficient roles because the conventional BEM yields unstable solutions.

Case study: To check the model's validity, a simplified case study was implemented. Consider an infinitely long, thin, conducting circular tube of radius $b$ which is split in two halves as shown in Fig. 3. The upper half is kept at a potential $V=V_{0}$ and the lower half at $V=-V_{0}$, respectively. To determine the electric potential distribution outside the tube, conventional BEM and the aforementioned regularization techniques were all tested.

(1) Analytical solution: Using the method of separation of variables with the Bessel functions [1], the desired electric potential distribution $V(r)$ in the space $r \geqslant b$ is

$$
V(r, \phi)=\left(\frac{4 V_{0}}{\pi}\right) \sum\left(\frac{1}{n}\right)\left(\frac{b}{r}\right)^{n}[\sin (n \phi)],
$$

where $\phi=\tan ^{-1}(y / x), r=\left(x^{2}+y^{2}\right)^{0.5}$ and $n$ is odd.

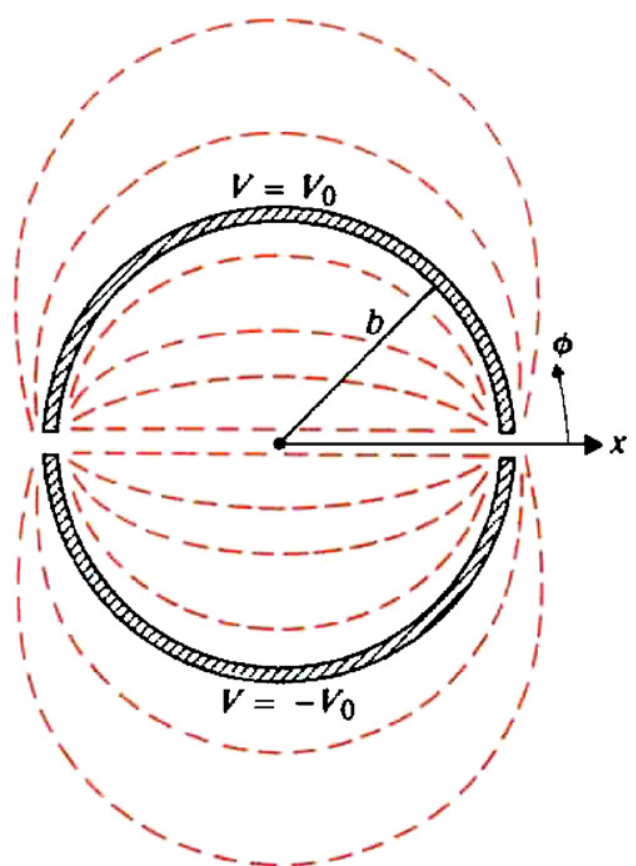

Fig. 3. Cross-section of split circular cylinder and equipotential lines.
(2) Conventional BEM without regularization techniques: In order to obtain the singularity from degenerate scale, the value of $b$ is assumed to be 1 , and four points will be analyzed using of BEM model (48 elements and 48 nodes), and compared with analytical data computed from Eq. (10).

First, the SVD technique was used to check the singular status of $[U]$ matrix. From Figs. 4 and 5, there is an abrupt drop of the reciprocal of condition number $\left(c^{-1}\right)$ and the first minimum singular value $\left(s_{1}\right)$ of $[U]$ matrix can be found if the radius of circle is close to 1 . Since $c^{-1}$ and $s_{1}$ of $[U]$ matrix are close to zero if the radius $b$ is 1 , this influence matrix $[U]$ is almost rank deficient and numerical results may be unstable. The results of electric potential were listed in Table 2. One can see from this table that the errors between analytical method and conventional BEM are very large because of singular matrix $[U]$. This is the reason why the regularization techniques presented in this article were needed to cope with degenerate problems. In addition, to investigate how seriously the rankdeficiency behaves, the second minimum singular value

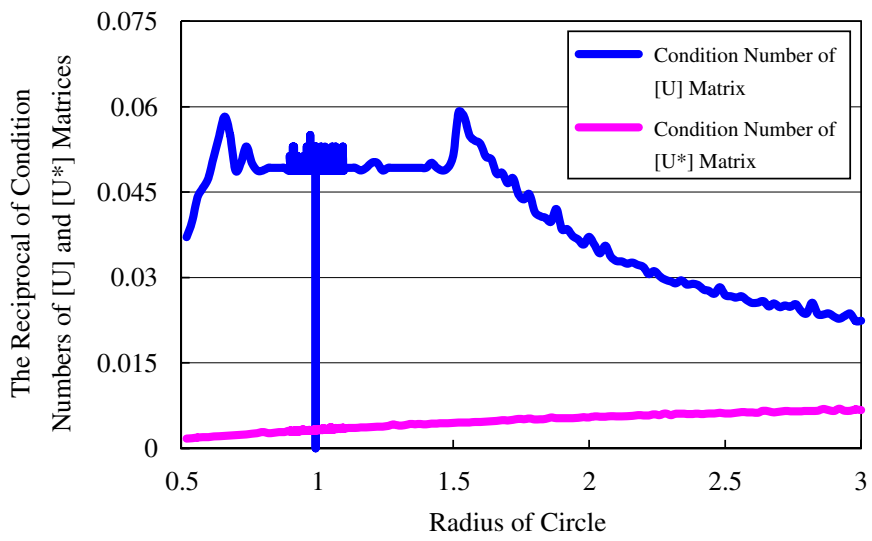

Fig. 4. The reciprocal of condition numbers vs. radius of circle using the $\mathrm{BEM}+\mathrm{SVD}$.

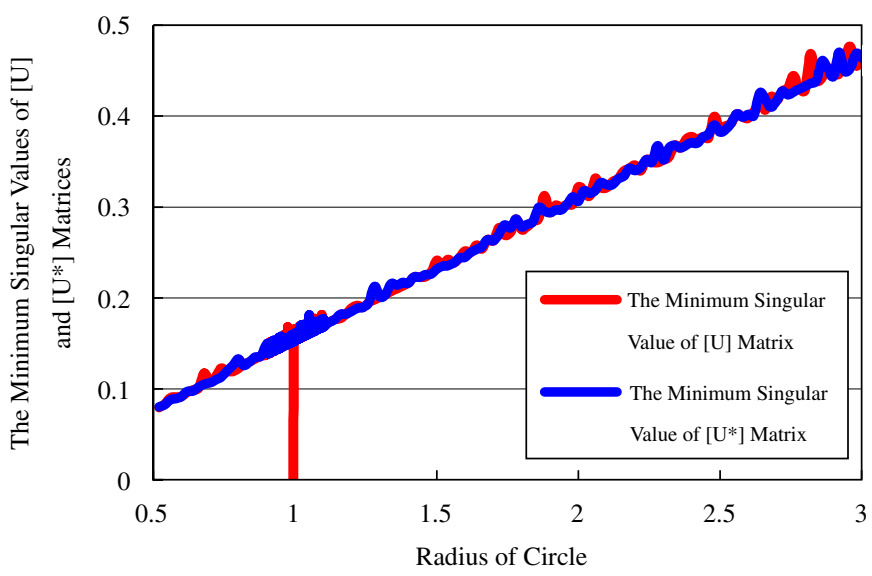

Fig. 5. The minimum singular values vs. radius of circle using the $\mathrm{BEM}+\mathrm{SVD}$. 
Table 2

The results of electric potential under conventional BEM and analytical methods

\begin{tabular}{llcr}
\hline $\begin{array}{l}\text { Locations }(x, y), \\
r=\left(x^{2}+y^{2}\right)^{0.5}\end{array}$ & $\begin{array}{l}\text { Results from } \\
\text { conventional BEM }\end{array}$ & $\begin{array}{l}\text { Results from } \\
\text { analytical method }\end{array}$ & $\begin{array}{l}\text { Error } \\
(\%)\end{array}$ \\
\hline$(17.5,15.0)$ & $0.10946 V_{0}$ & $0.03604 V_{0}$ & 203.68 \\
$(15.0,20.0)$ & $0.11615 V_{0}$ & $0.04083 V_{0}$ & 184.49 \\
$(12.5,30.0)$ & $0.11769 V_{0}$ & $0.03622 V_{0}$ & 224.91 \\
$(18.2,43.9)$ & $0.06563 V_{0}$ & $-0.02479 V_{0}$ & -364.71 \\
\hline
\end{tabular}

$\left(s_{2}\right)$ vs. radius $b$ of the circle was shown in Fig. 6. From this figure, $s_{2}$ of $[U]$ matrix was nonzero even the radius of circle is close to 1 . Hence the rank is deficient by one only, and this supports that only one CHIEF point is sufficient for this case.

(3) $B E M+R B M$ : In this case, a rigid body term $\beta(=1.0)$ was added first of all. The SVD technique was also used to check the singular status of $\left[U^{*}\right]$ matrix. Unlike $[U]$ matrix, there is no abrupt drop of $c^{-1}$ and $s_{1}$ of $\left[U^{*}\right]$ matrix can be found even the radius of circle is close to 1 (see Figs. 4 and 5). Since $c^{-1}$ and $s_{1}$ of $\left[U^{*}\right]$ matrix are nonzero whatever the radius $b$ is, this new influence matrix $\left[U^{*}\right]$ is nonsingular. The results of electric potential using $\left[U^{*}\right]$ were listed in Table 3 . The errors between analytical method and BEM + RBM shown in this table are less than $0.2 \%$. Therefore, BEM + RBM used in this article is a very efficient and easy numerical method for the electrostatic problems with singularity from a degenerate scale.

(4) BEM+CHIEF method: In this case, the CHIEF method was chosen because of exterior problem and an independent constraint was added to promote the rank of $[U]$. The SVD technique was used to decompose the $[U]$ matrix. From Fig. 7, there is no abrupt drop of $s_{1}$ for any radius $b$ if using CHIEF method. Since $s_{1}$ of $[U]$ matrix is nonzero, this new influence matrix $[U]$ is nonsingular. The results of electric potential were listed in Table 4. From this table, it is noted that the errors between analytical method and BEM + CHIEF method are less than $4.7 \%$. This concludes that BEM $+\mathrm{CHIEF}$ used in this article can also work well.

(5) Hypersingular formulation: Though $[U]$ matrix of the singular boundary integral equation is singular, the SVD technique was also used to check the singular status of $[L]$ matrix of the hypersingular boundary integral equation. If matrix $[L]$ is also singular, the regularization technique cannot be used in this case. From Fig. 8, no abrupt drop of $c^{-1}$ matrix is found if using hypersingular formulation even the radius of circle is close to 1 . By way of SVD, $c^{-1}$ of $[L]$ matrix is nonzero, and this influence matrix $[L]$ is nonsingular. The results of electric potential using $[L]$ were listed in Table 5. On comparison of the results for hypersingular formulation and analytical method (Eq. (10)), it can be seen that the errors between analytical method and hypersingular formulation are less than $1.6 \%$.

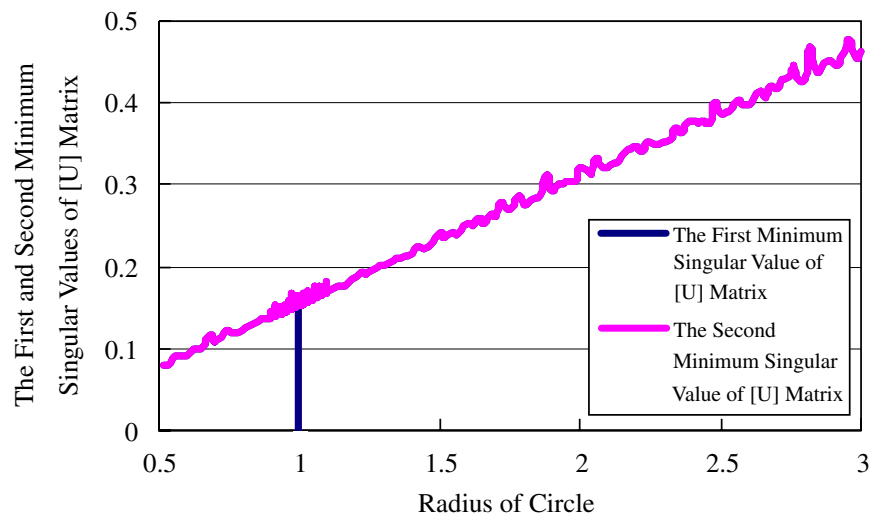

Fig. 6. The first and second minimum singular values vs. radius of circle using the BEM + SVD.

Table 3

The results of electric potential under $\mathrm{BEM}+\mathrm{RBM}$ and analytical methods

\begin{tabular}{lccc}
\hline $\begin{array}{l}\text { Locations }(x, y), \\
r=\left(x^{2}+y^{2}\right)^{0.5}\end{array}$ & $\begin{array}{l}\text { Results from } \\
\text { BEM + RBM }\end{array}$ & $\begin{array}{l}\text { Results from } \\
\text { analytical method }\end{array}$ & $\begin{array}{l}\text { Error } \\
(\%)\end{array}$ \\
\hline$(17.5,15.0)$ & $0.03599 V_{0}$ & $0.03604 V_{0}$ & -0.16 \\
$(15.0,20.0)$ & $0.04077 V_{0}$ & $0.04083 V_{0}$ & -0.14 \\
$(12.5,30.0)$ & $0.03617 V_{0}$ & $0.03622 V_{0}$ & -0.15 \\
$(18.2,43.9)$ & $-0.02479 V_{0}$ & $-0.02479 V_{0}$ & -0.02 \\
\hline
\end{tabular}

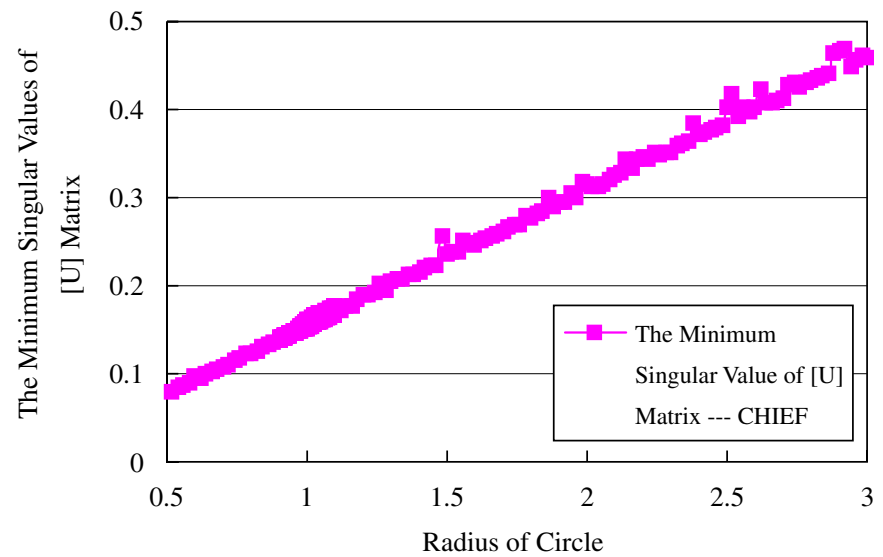

Fig. 7. The minimum singular values vs. radius of circle using the $\mathrm{BEM}+\mathrm{CHIEF}$.

Table 4

The results of electric potential under BEM + CHIEF and analytical methods

\begin{tabular}{lccr}
\hline $\begin{array}{l}\text { Locations }(x, y), \\
r=\left(x^{2}+y^{2}\right)^{0.5}\end{array}$ & $\begin{array}{l}\text { Results from } \\
\text { BEM + CHIEF }\end{array}$ & $\begin{array}{l}\text { Results from } \\
\text { analytical method }\end{array}$ & $\begin{array}{l}\text { Error } \\
(\%)\end{array}$ \\
\hline$(17.5,15.0)$ & $0.03692 V_{0}$ & $0.03604 V_{0}$ & 2.43 \\
$(15.0,20.0)$ & $0.04173 V_{0}$ & $0.04083 V_{0}$ & 2.21 \\
$(12.5,30.0)$ & $0.03721 V_{0}$ & $0.03622 V_{0}$ & 2.72 \\
$(18.2,43.9)$ & $-0.02364 V_{0}$ & $-0.02479 V_{0}$ & -4.64 \\
\hline
\end{tabular}




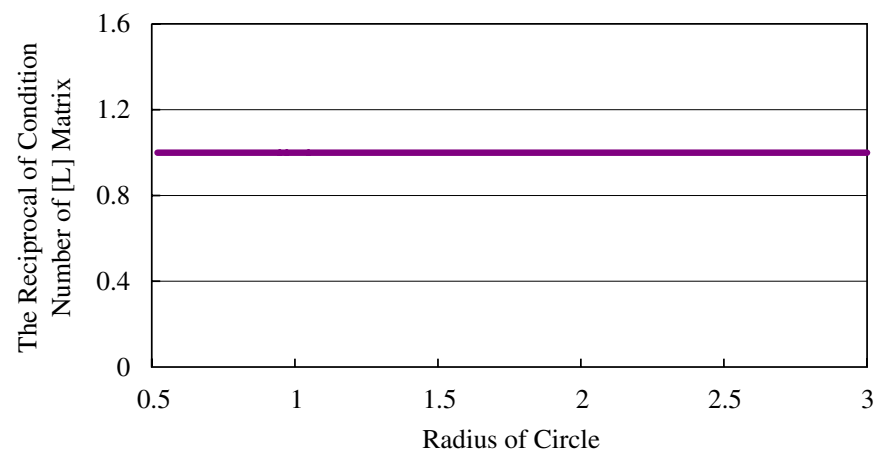

Fig. 8. The reciprocal of condition numbers vs. radius of circle using hypersingular formulation.

Table 5

The results of electric potential under hypersingular formulation and analytical methods

\begin{tabular}{llll}
\hline $\begin{array}{l}\text { Locations }(x, y), \\
r=\left(x^{2}+y^{2}\right)^{0.5}\end{array}$ & $\begin{array}{l}\text { Results from } \\
\text { BEM + hypersingular } \\
\text { formulation }[L],[M]\end{array}$ & $\begin{array}{l}\text { Results from } \\
\text { analytical } \\
\text { method }\end{array}$ & $\begin{array}{l}\text { Error } \\
(\%)\end{array}$ \\
\hline$(17.5,15.0)$ & $0.03550 V_{0}$ & $0.03604 V_{0}$ & -1.50 \\
$(15.0,20.0)$ & $0.04023 V_{0}$ & $0.04083 V_{0}$ & -1.46 \\
$(12.5,30.0)$ & $0.03568 V_{0}$ & $0.03622 V_{0}$ & -1.49 \\
$(18.2,43.9)$ & $-0.02450 V_{0}$ & $-0.02479 V_{0}$ & -1.20 \\
\hline
\end{tabular}

Therefore, hypersingular formulation used in this article also work very efficiently.

\section{Results and discussions}

(1) From the results of the electric potential shown in Tables 2-5 and Figs. 4-8, one can find that the conventional BEM without regularization techniques cannot solve the electrostatic problems with singularity arising from degenerate scale because the $[U]$ matrix of the singular boundary integral equation is singular. The regularization techniques presented in this article can analyze the electrostatic problems with degenerate scale, and the errors between the regularization techniques and analytical method are very small.

(2) For some special geometry, $[U]$ matrix of conventional BEM may be either singular or else numerically very close to singular. In this case, SVD plays a very important role. Not only it can diagnose the degenerate problem, it can also solve it and provides scientists and engineers a useful numerical answer.

(3) Basically only one CHIEF point is required to deal with the degenerate scale problem efficiently since the rank of $[U]$ matrix is deficient by only one if using the singular boundary integral equation. Nevertheless, the $[L]$ matrix of the hypersingular boundary integral equation is never singular whatever the radius is, and the hypersingular formulation (LM equation) can shift the zero singular value in the singular formulation (UT equation).
(4) To our best knowledge, it is very important to utilize SVD technique first to check the singular status of influence matrices before the BEM was used. If $[U]$ matrix is nonsingular, either convention BEM or DBEM can be used, otherwise the regularization techniques need to be used.

\section{Conclusions}

The regularization techniques, RBM, CHIEF and hypersingular formulation, of electrostatic problems with singularity arising from degenerate scale have been presented in this article. Comparisons of results between conventional BEM, regularization techniques and analytical analyses were discussed in order to demonstrate the superiority of regularization BEM in conjunction with SVD technique. It is concluded that the regularization techniques are particularly suitable for the electrostatic problem with singularity arising from a degenerate scale.

\section{Acknowledgment}

The assistance concerning SVD technique from Dr. I-Lin Chen (Department of Naval Architecture, Kaohsiung Institute of Marine Technology) is grateful.

\section{References}

[1] D.K. Cheng, Field and Wave Electromagnetics, Addison-Wesley Publishing Company, Inc., Reading, MA, 1989.

[2] M.N.O. Sadiku, Numerical Techniques in Electromagnetics, CRC Press, Inc., Boca Raton, FL, 1992.

[3] D.C. Liu, On the theory of the modal expansion method for the EM fields in side a closed volume, J. Phys. D: Appl. Phys. 36 (2003) 1629-1633.

[4] P.P. Silvester, F.L. Ferrari, Finite Elements for Electrical Engineers, Cambridge University Press, Cambridge, 1983.

[5] R.F. Harrington, T.K. Sarkar, Boundary elements and the method of moments, in: C.A. Brebbia, et al. (Eds.), Boundary Elements, CML Publ., Southampton, 1983, pp. 31-40.

[6] S.D. Senturia, N. Aluru, J. White, Simulating the behavior of MEMS devices: computational methods and needs, IEEE Comput. Sci. Eng. Magn. (1997) 30-43.

[7] S.W. Chyuan, Y.S. Liao, J.T. Chen, An efficient method for solving electrostatic problems,, IEEE Comput. Sci. Eng. Magn. (2003) 52-58.

[8] J.T. Chen, H.K. Hong, Review of dual boundary element methods with emphasis on hypersingular integrals and divergent series, Appl. Mech. Rev. ASME 52 (1) (1999) 17-33.

[9] S. Christiansen, Integral equations without a unique solution can be made useful for solving some plane harmonic problems, J. Inst. Math. Appl. 16 (1975) 143-159.

[10] R.F. Harrington, K. Pontoppidan, P. Abrahamsen, N.C. Albertsen, Computation of Laplacian potentials by an equivalent-source method, Proc. IEE 116 (10) (1969) 1715-1720.

[11] J.T. Chen, J.H. Lin, S.R. Kuo, Y.P. Chiu, Analytical study and numerical experiments for degenerate scale problems in boundary element method using degenerate kernels and circulants, Eng. Anal. Bound. Elem. 25 (2001) 819-828.

[12] M. Vable, Importance and use of rigid body mode in boundary element method, Int. J. Numer. Methods Eng. 29 (1990) 453-472.

[13] H.A. Schenck, Improved integral formulation for acoustic radiation problems, J. Acoust. Soc. Am. 55 (1) (1968) 41-58. 
[14] I.L. Chen, J.T. Chen, S.R. Kuo, M.T. Liang, A new method for true and spurious eigensolutions of arbitrary cavities using the combined Helmholtz exterior integral equation method, J. Acoust. Soc. Am. 109 (3) (2001) 982-998.

[15] G.H. Golub, C. Reinsch, Singular value decomposition and least square solutions, Numer. Math. 14 (1970) 403-420.

[16] M.A. Jaswon, G.T. Symm, Integral Equation Methods in Potential Theory and Electrostatics, Academic Press, New York, 1977.
[17] J.T. Chen, S.R. Lin, On the rank-deficiency problems in boundary integral formulation using the Fredholm alternative theory and singular value decomposition technique, in: Proceedings of Fifth World Congress on Computational Mechanics, Vienna, Austria, 7-12 July 2002.

[18] S. Christiansen, Detecting non-uniqueness of solutions to biharmonic integral equations through SVD, J. Comput. Appl. Math. 134 (2001) $23-35$. 